\title{
Prognostic significance of ST3GAL-1 expression in patients with clear cell renal cell carcinoma
}

Qi Bai ${ }^{1+}, \mathrm{Li} \mathrm{Liu}^{1+}$, Yu Xia ${ }^{1+}$, Qilai Long ${ }^{1}$, Jiajun Wang ${ }^{1}$, Jiejie X ${ }^{2^{*}}$ and Jianming Guo ${ }^{1^{*}}$

\begin{abstract}
Background: Aberrant sialylated carbohydrate synthesis is frequently noted in various cancers. Sialyltransferase ST3GAL-1, which adds a sialic acid in an a-2,3 linkage to Gal $\beta 1,3$ GalNAc, preforms an important role in modulating cellular behaviors. However, little is known about prognostic significance of ST3GAL-1 in clear cell renal cell carcinoma (ccRCC). In this study, we aimed to investigate the prognostic significance of sialyltransferase ST3GAL-1 and its correlation with clinical outcomes in patients with ccRCC.
\end{abstract}

Methods: A total of 286 patients who underwent nephrectomy between 2005 and 2007 in a single academic center were recruited. Immunohistochemical staining was performed on tissue microarrays to assess the expression level. Kaplan-Meier method and Cox proportional hazard model were applied to assess the prognostic value of ST3GAL-1. Nomograms were generated as prediction model for overall survival and disease free survival at 5 and 8 years after nephrectomy.

Results: The present results show high expression of ST3GAL-1 is associated with reduced overall survival ( $p=0.013$ ) and disease free survival $(p=0.004)$. In multivariate cox analyses, ST3GAL-1 was defined as an independent prognostic factor for overall survival $(p=0.006)$ and disease free survival $(p=0.001)$. After incorporation into the University of California Integrated Staging System (UISS) intermediate/high risk group for non-metastatic cCRCC, ST3GAL-1 could further distinguish patient with dismal prognosis ( $p=0.015$ and 0.002 for OS and DFS respectively). The nomograms revealed better predictive accuracy in predicting 5 - and 8 - year overall survival and disease free survival than the TNM stage alone.

Conclusions: ST3GAL-1 is an independent adverse prognostic factor for recurrence and survival of patients with CCRCC.

Keywords: Clear cell renal cell carcinoma, $\beta$-Galactoside a-2,3-Sialyltransferase 1, Prognosis, Nomogram, Overall survival, Disease free survival

\section{Background}

Renal cell carcinoma (RCC) is the most common solid lesion within kidney and accounts for $2-3 \%$ of all cancers in adults [1]. The predominant histologic subtype is clear cell renal cell carcinoma (ccRCC) (70-85 \%) [2]. Although most patients with localized tumors can be cured with surgical therapies, $20-30 \%$ of the patients

\footnotetext{
* Correspondence: jjxufdu@fudan.edu.cn; guo.jianming@zs-hospital.sh.cn ${ }^{\dagger}$ Equal contributors

${ }^{2}$ Department of Biochemistry and Molecular Biology, School of Basic Medical Sciences, Fudan University, Shanghai 200032, China

'Department of Urology, Zhongshan Hospital, Fudan University, Shanghai 200032, China
}

without any evidence of metastasis will develop relapse and/or metastatic RCC in the future [3]. Currently, TNM stage, Fuhrman grade, and Eastern Cooperative Oncology Group performance status (ECOG PS) have been widely used as predictors of clinical outcomes for patients with RCC. Several outcomes prognostic prediction algorithms like University of California Integrated Staging System (UISS), which incorporates TNM stage, Fuhrman grade, and ECOG PS, have been proposed previously to stratify the clinical outcomes after nephrectomy $[4,5]$. But the biological nature of ccRCC is kind of complex with an unpredictable course; even tumors 
with the comparable stage or the same pathological type could show a wide variation in biological behavior and clinical outcomes. The improvement of the predictors of clinical outcomes for RCC is needed [6,7].

Change in the structure of glycan chains is one of the most shared features of the malignant transformation [8]. The oligosaccharide chains of glycoprotein and glycolipid are often terminated by sialic acids, which play intensely important role in regulating physiologically and pathologically interactions with ligands [9]. In types of cancers, abnormal high level of sialylated tumor associated carbohydrate antigens are frequently noted $[10,11]$. The upregulated sialylation may mediate key pathophysiological events during various steps of tumor progression [12-14]. $\beta$-galactoside $\alpha$-2,3-sialyltransferase 1 (ST3GAL-1), which catalyzes the addition of sialic acid in an $\alpha-2,3$ linkage to Gal $\beta 1,3$ GalNAc, preforms important role in modulating cellular behavior. Increasing studies have revealed that the abnormal activity of ST3GAL-1 contributes to the tumor progression [10, 15-17]. However, few studies dedicated to evaluate the expression of ST3GAL-1 and its correlation with ccRCC pathological characters, making the issue elusive.

In this study, we aimed to investigate the prognostic significance of sialyltransferase ST3GAL-1 expression and its correlation with clinical outcomes in patients with ccRCC. ST3GAL-1 expression was assessed by immunohistochemistry, and nomograms integrating ST3GAL-1 with other prognostic parameters were generated to refine individual risk stratification in ccRCC patients.

\section{Methods}

\section{Patients}

We retrospectively collected 286 patients who underwent nephrectomy between 2005 and 2007 at Zhongshan Hospital Fudan University (Shanghai, China). Patient records include TNM stage, Fuhrman grades, histology type, ECOG PS were extracted from the database of the institution, and all the data above were accessible. Other clinicopathological and baseline demographic characters include age, sex, tumor size, and necrosis were also collected retrospectively. Patients with localized RCC $(n=271)$ were treated with either nephron spare surgery or radical nephrectomy. Patients with metastatic RCC $(n=15)$ at diagnosis received cytoreductive nephrectomy followed by interferon- $\alpha$ based immunotherapy. Tumor stage and postoperative histopathological type was determined according to the 2010 AJCC TNM classification [18]. The inclusion criteria were as follows: (1) the histopathological type proved to be ccRCC, (2) all the individuals had no history of anticancer therapy before the nephrectomy, and (3) had no history of other malignant before. If the histopathology was mostly necrosis $(>80 \%)$ or the morphologic features represent a mixture type of clear cell RCC and other RCC were excluded from the present study. The UISS predictive model was applied to all cases to stratify the risk groups. Most patients underwent regular follow-up every 6 months or earlier for the first 2 years right after the nephrectomy and every 12 months thereafter. Clinical Research Ethics Committee of Zhongshan Hospital, Fudan University had approved the study and granted permissions to access the patient records. Written, informed consent was obtained from each individual enrolled in the study.

\section{Immunohistochemical staining and evaluation}

Hematoxylin and eosin-stained slides had been screened for optimal tumor content before we constructed tissue microarray (TMA) slides. Two cores were taken from the formalin-fixed, paraffin embedded surgical specimens with $2.0 \mathrm{~mm}$ diameter punch to make sure each array represented the dominant focus of ccRCC. Immunohistochemical staining was performed on TMA, and the primary antibody was rabbit polyclonal anti-SIAT4A antibody (ab96129, Abcam, Cambridge, MA, USA). The images of stained tissue were captured by the computerized image system composed of an Olympus CCD camera connected to a Nikon eclipse Ti-s microscope. The immunohistochemistry samples were scanned at high-

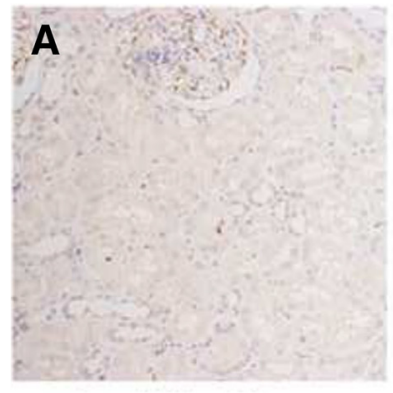

Normal Kidney Tissue

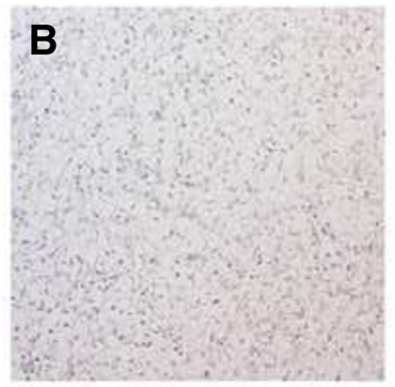

ST3GAL-1 Low Expression

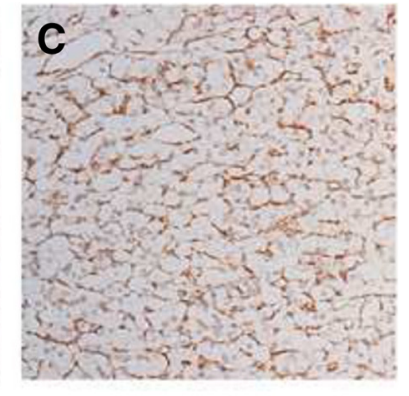

ST3GAL-1 High Expression

Fig. 1 Representative photographs of ST3GAL-1 immunostaining. ST3GAL-1 expression in normal kidney tissue (a); Low ST3GAL-1 expression in tumor tissue (b); High ST3GAL-1 expression in tumor tissue (c). Original magnification: $\times 200$ 
power magnification $(\times 200)$ and photographed by NISElements F3.2 software. The staining intensity and extent were scored by two independent pathologists who were blind to the clinical outcomes using the integrated optical density (IOD) calculated by Image-Pro Plus version 6.0 software (Media Cybernetics Inc., Bethesda, MD, USA). The intensity score was graded as 0 (negative), 1 (weak), 2 (moderate), and 3 (strong); the extent score was calculated by the percentage of the positive cells $(0-100 \%)$. The staining intensity and extent were then multiplied to generate the expression score ranging from 0 to 300 . The score of 210 was selected as the cutoff point of high/low expression by the X-Tile software (Yale University School of Medicine, New Haven, CT, USA).

\section{Statistical analyses}

The correlation between ST3GAL-1 expression and clinicopathological characteristics was analyzed with Fisher's exact test, $\mathrm{X} 2$ test or $\mathrm{t}$ test. Kaplan-Meier method was applied to compare overall survival (OS) and disease free survival (DFS) for groups of low and high expression. Statistical significance was calculated using log-rank test. Multivariate Cox proportional hazard models were used to estimate and test the impact of demographic characteristics, clinical features and ST3GAL-1 expression on overall survival and disease free survival. Hazard ratio (HR) and $95 \%$ confidence interval (CI) were calculated.

The nomograms were generated using $\mathrm{R}$ software with "rms" package (R Foundation for Statistical Computing, Vienna, Austria). Selection of variables included in nomograms was based on statistical significance of multivariate analyses, and tumor size was also included in the model as continuous variable. For DFS, we divided T stage variable into $\mathrm{T} 1 \mathrm{a}$ and $\mathrm{T} 1 \mathrm{~b}+\mathrm{T} 2$, due to the clinical similarity for metastases after radical nephrectomy [19]. Calibration plots for 5- and 8- year OS and DFS were generated to explore the performance characteristics of the predictive model. The Harrell's concordance indices (c-indices) were used to measure the prognostic accuracy. All data analyses above were performed using SPSS version 19.0 (SPSS Inc., IL, Chicago, USA) and R software with "rms" package (R Foundation for Statistical Computing, Vienna, Austria). All statistical tests were two sided and considered significant at two-sided $p<0.05$ levels.

\section{Results}

ST3GAL-1 expression and survival analyses in human ccRCC The representative images of immunostaining were present in Fig. 1. The ST3GAL-1 expression was very low or not detectable in normal kidney tissues (Fig. 1a). The positive staining of ccRCC predominantly appeared in the cell cytoplasm (Fig. 1c). We analyzed a total of 286 patients in the present study. As Table 1 presented, the mean (SD) age was 55.0 (13.0) years old and the median follow up was 99
Table 1 Associations between ST3GAL-1 expression and clinicopathological characteristics in patients with cCRCC

\begin{tabular}{|c|c|c|c|c|}
\hline \multirow[b]{2}{*}{ Characteristics } & \multirow[b]{2}{*}{ All patients } & \multicolumn{3}{|c|}{ ST3gal-1 expression } \\
\hline & & Low & High & $p^{*}$ \\
\hline Age (years) & & & & 0.656 \\
\hline Mean \pm SD & $55.0 \pm 13.0$ & $54.7 \pm 13.6$ & $55.4 \pm 11.9$ & \\
\hline Gender & & & & 0.455 \\
\hline Female & 90 & 57 & 33 & \\
\hline Male & 196 & 115 & 81 & \\
\hline T stage & & & & 0.913 \\
\hline $\mathrm{T} 1$ & 180 & 106 & 74 & \\
\hline $\mathrm{T} 2$ & 24 & 14 & 10 & \\
\hline T3 & 79 & 50 & 29 & \\
\hline T4 & 3 & 2 & 1 & \\
\hline N stage & & & & 1.000 \\
\hline NO & 284 & 171 & 113 & \\
\hline N1 & 2 & 1 & 1 & \\
\hline M stage & & & & 0.102 \\
\hline MO & 273 & 167 & 106 & \\
\hline M1 & 13 & 5 & 8 & \\
\hline TNM stage & & & & 0.334 \\
\hline 1 & 176 & 105 & 71 & \\
\hline$\|$ & 20 & 12 & 8 & \\
\hline III & 75 & 49 & 26 & \\
\hline IV & 15 & 6 & 9 & \\
\hline $\begin{array}{l}\text { Fuhrman nuclear } \\
\text { grade grade }\end{array}$ & & & & 0.604 \\
\hline 1 & 31 & 19 & 12 & \\
\hline 2 & 216 & 127 & 89 & \\
\hline 3 & 37 & 24 & 13 & \\
\hline 4 & 2 & 2 & 0 & \\
\hline Necrosis & & & & 0.652 \\
\hline Absent & 249 & 151 & 98 & \\
\hline Present & 37 & 21 & 16 & \\
\hline ECOG PS & & & & 0.587 \\
\hline 0 & 198 & 117 & 81 & \\
\hline$\geq 1$ & 88 & 55 & 33 & \\
\hline
\end{tabular}

Abbreviations: ECOG PS Eastern Cooperative Oncology Group performance status, cCRCC clear cell renal cell carcinoma

${ }^{*} \mathrm{X} 2$ test or $\mathrm{t}$ test was performed. $p<0.05$ was regard as statistically significant

(range 2.63-120.47) months. Thirteen patients had distant metastasis, and two patients had regional lymph node metastasis at time of surgery. The Kaplan-Meier curves show that patients with high ST3GAL-1 expression level tend to have significantly adverse outcomes for OS $(p=0.013$, Fig. 2a) than those with low ST3GAL-1 expression patients. For patients with localized ccRCC, the differences 

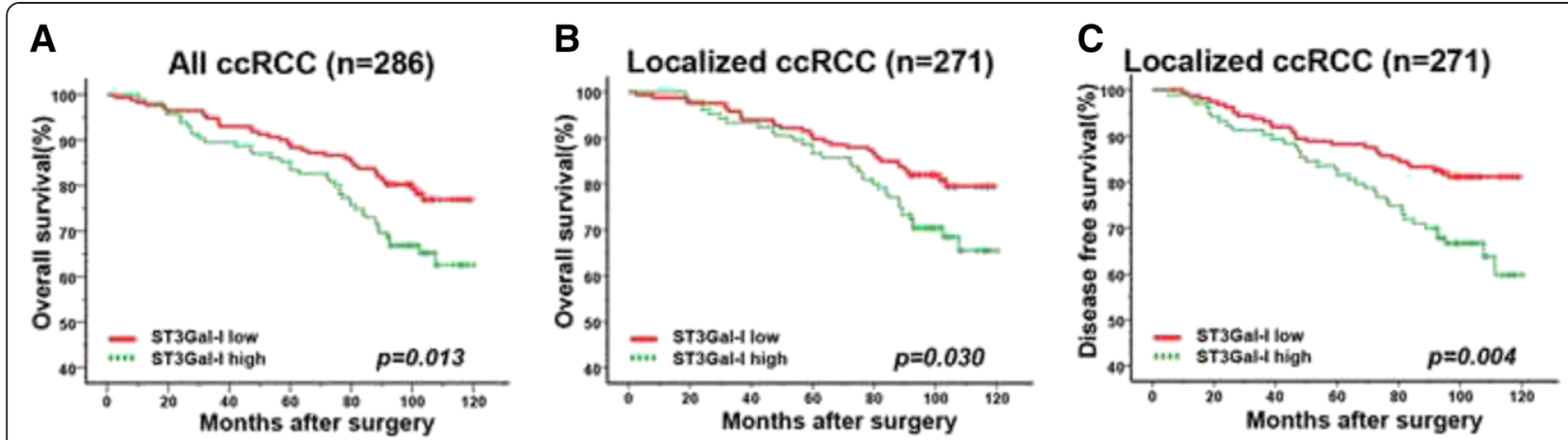

Fig. 2 Kaplan-Meier analyses for overall survival and disease free survival of patients with cCRCC according to ST3GAL-1 expression. Overall survival according to ST3GAL-1 expression in all CCRCC patients (a); overall survival according to ST3GAL-1 expression in localized ccRCC patients (b); disease free survival according to ST3GAL-1 expression in localized ccRCC patients (c); p-value was calculated by Log rank test, $<0.05$ was regarded as statistically significant

were still significant in both OS $(p=0.030$, Fig. $2 \mathrm{~b})$ and DFS $(p=0.004$, Fig. 2c).

\section{Extension of the UISS (non-metastatic) prognostic model} with ST3GAL-1

The UISS (non-metastatic) algorithm performed well in stratifying the risk group for overall survival and disease free survival in our ccRCC cohort $(p<0.001)$. Furthermore, we sought to investigate whether incorporation of high/low ST3GAL-1 expression into UISS outcome algorithm would improve the predictive accuracy by calculating c-index. In the IR/HR group for localized ccRCC, significant difference was found in OS $(p=0.015)$ and DFS $(p=0.002)$ according to the ST3GAL-1 expression (Fig. 3b, d), however ST3GAL-1 failed to further stratify patients with different prognosis in UISS LR patients (Fig. 3a, c). The c-indices of
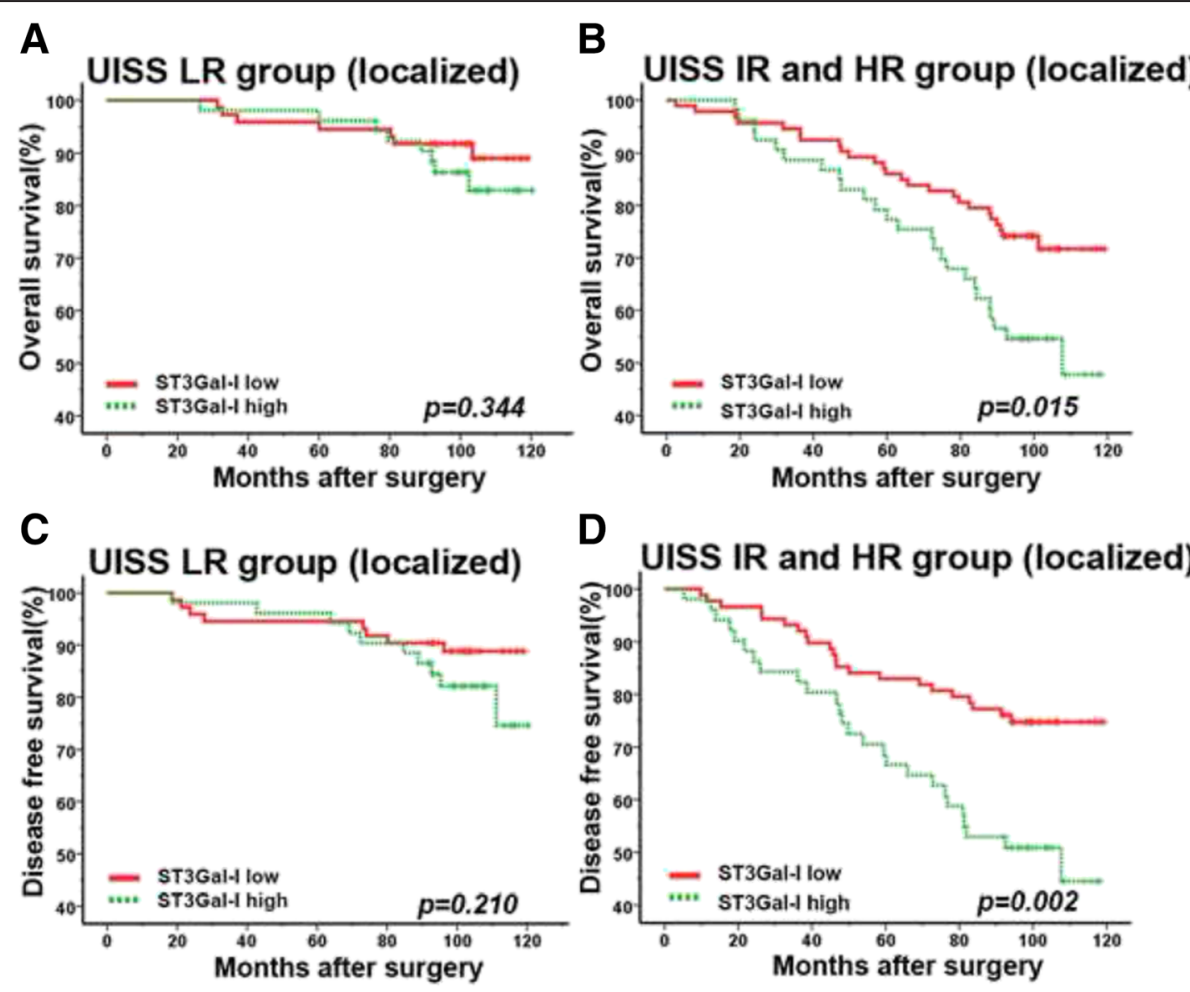

Fig. 3 Kaplan-Meier analyses for overall survival and disease free survival of patients in UISS (non-metastatic) subgroups. Overall survival for patients in the UISS (non-metastatic) low risk group (a) and intermediate/high risk group (b) according to ST3GAL-1 expression; disease free survival for patients in the UISS (non-metastatic) low risk group (c) and intermediate/high risk group (d) according to ST3GAL-1 expression; UISS: University of California LoS Angeles Integrated Staging System; $p$-value was calculated by Log rank test, $<0.05$ was regarded as statistically significant 
the UISS were 0.65 and 0.63 for OS and DFS respectively, and improved to 0.69 and 0.68 when ST3GAL-1 was added. For patients with metastatic ccRCC, we didn't use ST3GAL-1 to further refine the UISS (metastatic) algorithm due to the limited individuals.

\section{Multivariate cox regression analyses}

To investigate the prognostic impact of ST3GAL-1 on ccRCC, multivariate Cox regression models were applied (Table 2). In the multivariate cox models, patients with higher ST3GAL-1 expression showed a significantly reduced OS $(p=0.006)$ and DFS $(p=0.001)$ compared with their counterparts. It was also confirmed that TNM stage $(p<0.001)$, Fuhrman grade $(p=0.044)$, Necrosis $(p=0.045)$, and ECOG PS $(p=0.047)$ were independent prognostic factors for OS, and T stage $(p=0.003)$, Fuhrman grade $(p=0.020)$ and necrosis $(p=0.015)$ were independent prognostic factors for DFS in ccRCC.

\section{Nomogram for predicting overall survival and disease free survival in ccRCC}

Nomograms were generated to predict OS (Fig. 4a) and DFS (Fig. 5a) at 5 and 8 years after nephrectomy. Total points were calculated to evaluate the clinical outcomes, with higher point indicating more adverse outcome probability. Calibration plots of the nomograms are shown for 5- and 8- year prediction of OS (Fig. 4b, c) and DFS (Fig. 5b,c). The Harrell's c-indices, which indicated the accuracy of the prognostic model, were 0.753 (95\% CI, 0.701-0.805) and 0.745 (95 \% CI 0.690-0.800) for OS and DFS respectively, higher than that of the TMN stage alone (0.703, $95 \%$ CI: $0.647-0.759$ and 0.660, 95 \% CI: 0.599-0.721).

\section{Discussion}

Glycosylation is quite common in posttranslational modifications. Almost all the cell surface proteins are glycosylated. The aberrant glycosylation, which is aroused by the altered expression and activity of glycosyltransferase, can dramatically affect the glycoprotein and then change the behavior of cell [20-22]. Sialic acids, a family of nine carbon sugars are one of the most important monosaccharide decorating the oligosaccharide chains of several classes of cell surface and secreted glycan molecules. The sialylation of the glycan plays very important role in regulating cellular interaction and signal transduction. The prominent position on cell membrane allows sialoglycan to effectively participate in cell-cell and cell-extracellular matrix interaction, including adhesion, migration, and immune recognition [23]. Hypersialylation of glycan in tumor cells is associated with metastatic behaviors including invasion and enhanced cell survival and correlates with a poor prognosis for patients with cancer [24].

It is now well-established that altered activity of sialyltransferase is one of the key mechanisms to trigger aberrant sialylation of glycans and expression of specific tumor associated carbohydrates in cancer [24]. However, researches focus on the sialylation glycans and sialyltransferase in ccRCC is now devoid. So in this study, we put our focus on the sialyltransferase ST3GAL-1, which adds a sialic acid in an $\alpha-2,3$ linkage to Gal $\beta 1,3$ GalNAc. In the study, we have revealed the dismal role of ST3GAL-1 and its impact on patients with ccRCC by survival analyses. The present results showed that ccRCC patients with higher level of ST3GAL-1 expression tend to have unfavorable clinical outcomes than the counterparts. We also demonstrated that incorporating ST3GAL-1 into UISS (non-metastatic) could refine the algorithm prediction. In multivariate cox regression model, it was proved

Table 2 Multivariate cox regression analyses for overall survival and disease free survival in ccRCC patients

\begin{tabular}{|c|c|c|c|c|c|c|}
\hline \multirow[t]{2}{*}{ Variables } & \multicolumn{3}{|c|}{ Overall survival } & \multicolumn{3}{|c|}{ Disease free survival } \\
\hline & $\overline{H R}$ & $95 \% \mathrm{Cl}$ & $p^{*}$ & $\mathrm{HR}$ & $95 \% \mathrm{Cl}$ & $p^{*}$ \\
\hline TNM stage & & & $<0.001$ & & & 0.003 \\
\hline$I I I+\mid \mathrm{IV}$ vs $I+\|$ & 2.76 & $1.75-4.34$ & & 2.13 & $1.30-3.51$ & \\
\hline Fuhrman & & & 0.044 & & & 0.020 \\
\hline $3+4$ vs $1+2$ & 1.85 & $1.02-3.37$ & & 2.19 & $1.13-4.23$ & \\
\hline Necrosis & & & 0.045 & & & 0.015 \\
\hline Present vs Absent & 1.81 & $1.01-3.25$ & & 2.16 & $1.16-4.00$ & \\
\hline ECOG PS & & & 0.047 & & & 0.267 \\
\hline$\geq 1$ vs 0 & 1.61 & $1.01-2.56$ & & 1.35 & $0.80-2.29$ & \\
\hline ST3GAL-1 & & & 0.006 & & & 0.001 \\
\hline High vs Low & 1.88 & $1.20-2.94$ & & 2.30 & $1.40-3.77$ & \\
\hline
\end{tabular}

Abbreviations: ECOG PS Eastern Cooperative Oncology Group performance status, $\mathrm{Cl}$ confidence interval, $H R$ hazard ratio *Data obtained from the Cox proportional hazards model; $p<0.05$ was regard as statistically significant 


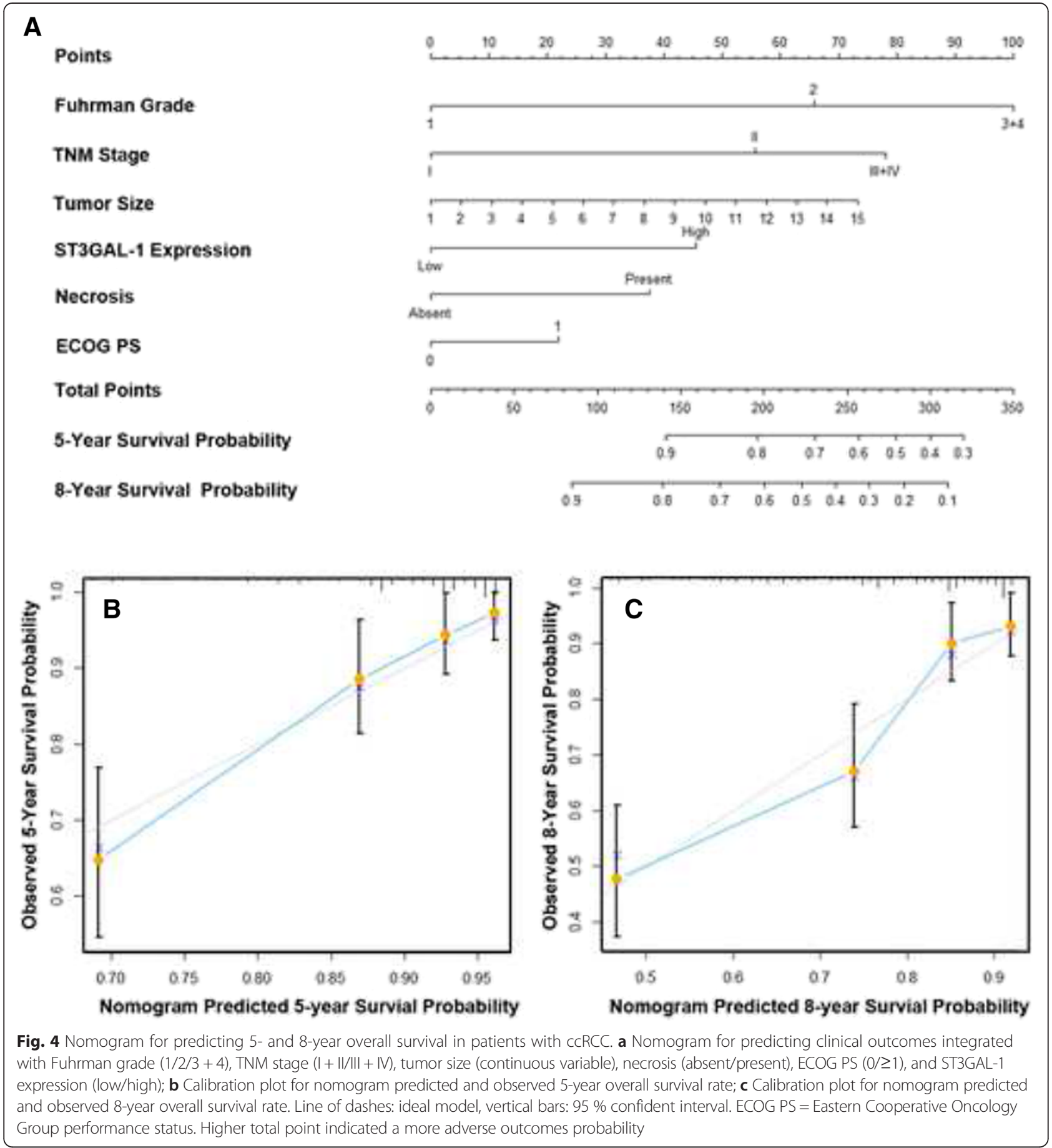

that ST3GAL-1 was an independent prognostic factor for $\mathrm{OS}$ and DFS. Unexpectedly, the $\mathrm{N}$ stage in multivariate analyses was not considered an independent prognostic factor, which was probably due to the limited number of N1 individuals $(n=2)$.

Several studies have elucidated the association of ST3GAL-1 with tumor progression. Overexpression of ST3GAL-1 has been reported in types of tumors like colon and breast cancers $[10,17]$. P. Videira found mRNA level of ST3GAL-1 was significantly higher in malignant bladder tumor [15]. It has also been confirmed that abnormal ST3GAL-1 activities could involve in malignant cell transformation and tumor progression. In breast carcinoma, constitutive ST3GAL-1 expression contributed to an unfavorable prognosis [16]. In colorectal carcinoma, ST3GAL-1 expression was associated with lymph node 
A

Points

T Stage

Fuhrman Grade

Tumor Size

ST3GAL-1 Expression

Necrosis

Total Points

5-Year DFS Probability

8-Year DFS Probability

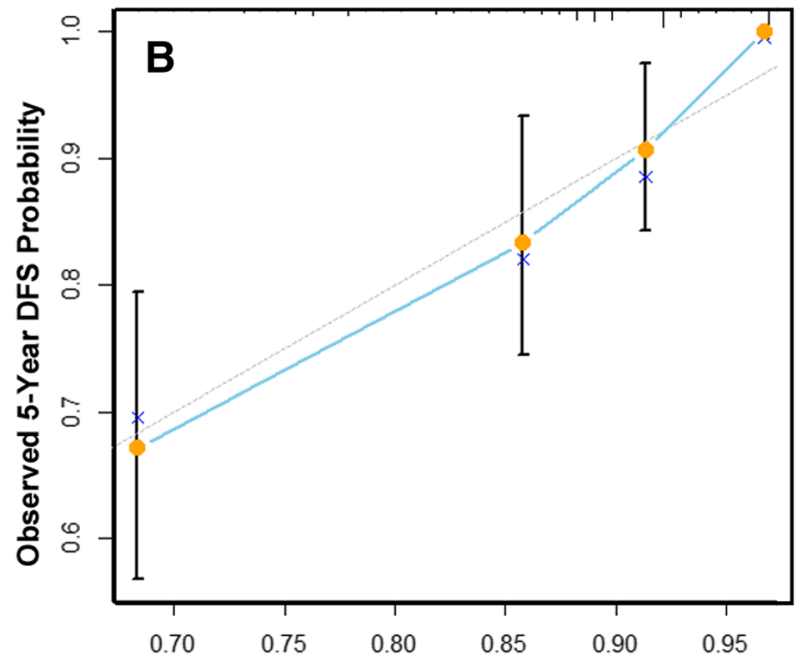

Nomogram Predicted 5-year DFS Probability
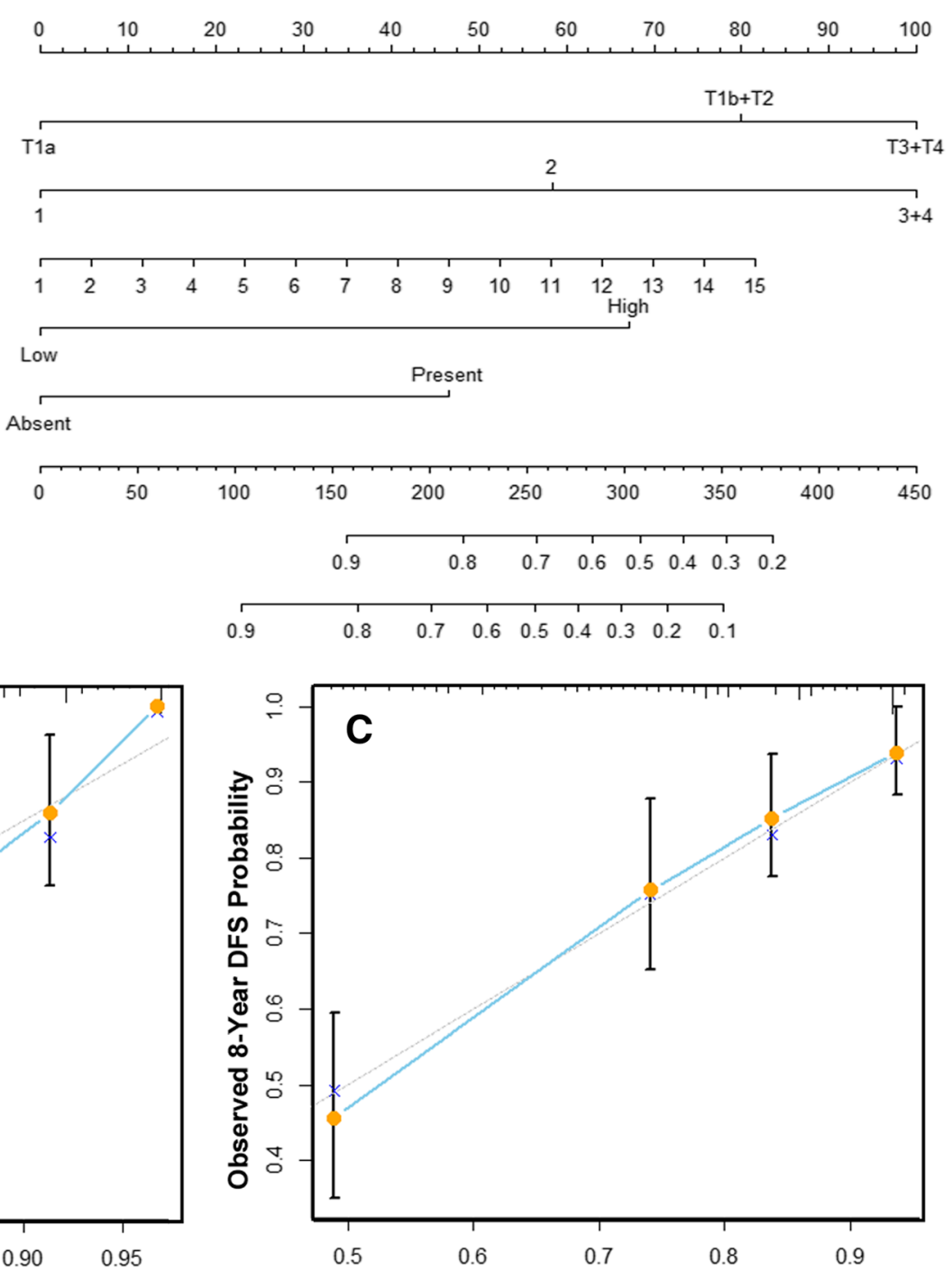

Nomogram Predicted 8-year DFS Probability

Fig. 5 Nomogram for predicting 5- and 8-year disease free survival in patients with ccRCC. a Nomogram for predicting disease free survival integrated with T stage (T1a/T1b + T2/T3 + T4), Fuhrman grade (1/2/3 + 4), tumor size (continuous variable), necrosis (absent/present) and ST3GAL-1 expression (low/high); b Calibration plot for nomogram predicted and observed 5-year disease free survival rate; c Calibration plot for nomogram predicted and observed 8-year disease free survival rate. Line of dashes: ideal model, vertical bars: $95 \%$ confident interval. Higher total point indicated a more adverse outcomes probability

metastasis [10]. Previous studies have reported that EMT, which is essential for tumor progression, is associated with altered expression of sialoglycans [25]. Upregulation of sialyltransferase and following expression of sialoglycan is an important step underlying the migratory phenotype during EMT. Moreover, the proto-oncogene, cmyc could up-regulate the ST3GAL-1, while the metastasis suppressor gene nm23-H1 plays an opposite role [25, 26], indicating the protumoral role of ST3Gal-1 in the development and progress of tumors.

Selectins, receptors for sialyl Lewis ${ }^{\mathrm{x}}\left(\mathrm{sLe}^{\mathrm{x}}\right)$ and sialyl Lewis $^{\mathrm{a}}\left(\mathrm{sLe}^{\mathrm{a}}\right)$ expressed on endothelial and blood cells play an important role in mediating malignant cells dissemination $[12,27]$. Recent reports have been indicative of ST3GAL-1's responsibility for the high expression of the sialoglycans $\operatorname{sLe}^{\mathrm{x}}$ and $\operatorname{sLe}^{\mathrm{a}}[25,28]$. Increased sLe $^{\mathrm{x}}$ was reported in liver metastasis of colorectal cancer, and was associated with poor patient survival and early recurrence in colorectal carcinoma [27, 29]. The interactions between selectins with $\mathrm{sLe}^{\mathrm{x} / \mathrm{a}}$ result in tumor cell adherence to blood vessels, thus facilitate extravasation into surrounding tissue, and trigger angiogenesis [30]. After tumors invade into vasculature, malignant cells with higher ST3GAL-1 were more likely to aggregate 
with platelets, and eventually lodge in the small vessels and distant organs [31]. Thus, it is conceivable that elevated sLe ${ }^{\mathrm{x} / \mathrm{a}}$ antigen synthesis caused by ST3Gal-1 in the tumor favors tumor cells to bind to selectins and then extricate from the primary tumor enter into blood stream and promote metastasis formation. This could give a possible explanation for our finding that patients with higher ST3GAL-1 expression are more likely to suffer recurrence and metastasis in the future, and ST3GAL-1 expression is also an independent risk factor for the disease free survival in ccRCC. Nowadays, increasing researches focusing on the sialylated glycan pathways have show a remarkable effectiveness for tumor therapy in preclinical and clinical studies [32-34], which might be suggestive that targeting sialyltransferase could be a novel approach to deal with RCC some day.

At present, the role of ST3GAL-1 in ccRCC is far from fully elucidation and need further exploration. Limitations of our study are the retrospective design and relatively small study cohort. A multicenter and prospective study is needed to validate the results. Moreover, other sialyltransferase like ST3GAL-3, ST3GAL-4 were not analyzed in the present study and merit further research.

\section{Conclusion}

In conclusion, we have confirmed ST3GAL-1 is correlated with unfavorable outcomes and could be used as an independent prognosticator in patients with ccRCC. We also developed nomograms for OS and DFS, which could give a better prediction for patients with ccRCC after surgery.

\section{Abbreviations \\ ST3GAL-1: $\beta$-Galactoside a-2,3-Sialyltransferase 1; RCC: Renal cell carcinoma; cCRCC: Clear cell renal cell carcinoma; OS: Overall survival; DFS: Disease free survival; UISS: University of California Integrated Staging System; \\ AJCC: American Joint Committee on Cancer; EMT: Epithelial-to-mesenchymal transition; TMA: Tissue microarray; C-index: Concordance index; \\ $\mathrm{Cl}$ : Confidence interval.}

\section{Competing interests}

The authors declare no conflict of interest.

\section{Authors' contributions}

QB carried out and conducted experiments, performed statistical analysis, and drafted the manuscript. LL participated in the collection of patient materials and drafting of the manuscript. YX carried out laboratory work and data analysis. QL participated in the study design and collection of related articles. JW performed laboratory work and participated in the correction of words in the manuscript. JX took charge of the study design and revising manuscript critically for important intellectual content. JG conceived of the study, and led the data analysis and oversaw the drafting of the manuscript. All authors read and approved the final manuscript.

\section{Acknowledgments}

This work was supported by grants from National Natural Science Foundation of China (31100629, 31270863, 81471621, 81472227 and 81472376), Program for New Century Excellent Talents in University (NCET-13-0146), Shanghai Health and Family Planning Commission (14ZR1406300) and Shanghai Rising-Star Program (13QA1400300). All these study sponsors have no roles in the study design, in the collection, analysis, and interpretation of data.
Received: 26 August 2015 Accepted: 3 November 2015

Published online: 09 November 2015

\section{References}

1. Siegel R, Ma JM, Zou ZH, Jemal A. Cancer statistics, 2014. Ca-Cancer J Clin. 2014;64(1):9-29.

2. Escudier B, Porta C, Schmidinger M, Algaba F, Patard JJ, Khoo V, et al. Renal cell carcinoma: ESMO Clinical Practice Guidelines for diagnosis, treatment and follow-up. Ann Oncol. 2014;25:49-56.

3. Stewart GD, O'Mahony FC, Powles T, Riddick ACP, Harrison DJ, Faratian D. What can molecular pathology contribute to the management of renal cell carcinoma? Nat Rev Urol. 2011;8(5):255-65.

4. Zisman A, Pantuck AJ, Wieder J, Chao DH, Dorey F, Said JW, et al. Risk group assessment and clinical outcome algorithm to predict the natural history of patients with surgically resected renal cell carcinoma. J Clin Oncol. 2002;20(23):4559-66.

5. Patard JJ, Kim HL, Lam JS, Dorey FJ, Pantuck AJ, Zisman A, et al. Use of the University of California Los Angeles integrated staging system to predict survival in renal cell carcinoma: An International Multicenter Study. J Clin Oncol. 2004;22(16):3316-22

6. Eichelberg C, Junker K, Ljungberg B, Moch H. Diagnostic and prognostic molecular markers for renal cell carcinoma: a critical appraisal of the current state of research and clinical applicability. Eur Urol. 2009;55(4):851-63.

7. Sun M, Shariat SF, Cheng C, Ficarra V, Murai M, Oudard S, et al. Prognostic factors and predictive models in renal cell carcinoma: a contemporary review. Eur Urol. 2011;60(4):644-61.

8. Hakomori SI. Aberrant glycosylation in tumors and tumor-associated carbohydrate antigens. Adv Cancer Res. 1989;52:257-331.

9. Harduin-Lepers A, Vallejo-Ruiz V, Krzewinski-Recchi MA, Samyn-Petit B, Julien S, Delannoy P. The human sialyltransferase family. Biochimie. 2001;83(8):727-37.

10. Schneider F, Kemmner W, Haensch W, Franke G, Gretschel S, Karsten U, et al. Overexpression of sialyltransferase CMP-sialic Acid: Gal beta 1,3GalNAc$R$ alpha 6-sialyltransferase is related to poor patient survival in human colorectal carcinomas. Cancer Res. 2001;61(11):4605-11.

11. Cohen AM, Allalouf D, Djaldetti M, Weigl K, Lehrer N, Levinsky H. Sialyltransferase activity in plasma-cells of multiple-myeloma. Eur J Haematol. 1989:43(3):191-4.

12. Dimitroff CJ, Pera P, Dall'Olio F, Matta KL, Chandrasekaran EV, Lau JTY, et al. Cell surface N-acetylneuraminic acid alpha 2,3-galactoside-dependent intercellular adhesion of human colon cancer cells. Biochem Bioph Res Co. 1999;256(3):631-6.

13. Ganzinger U, Deutsch E. Serum sialyltransferase levels as a parameter in the diagnosis and follow-up of gastrointestinal tumors. Cancer Res. 1980;40(4):1300-4.

14. Schultz MJ, Swindall AF, Bellis SL. Regulation of the metastatic cell phenotype by sialylated glycans. Cancer Metast Rev. 2012;31(3-4):501-18.

15. Videira PA, Correia M, Malagolini N, Crespo HJ, Ligeiro D, Calais FM, et al. ST3Gal.I sialyltransferase relevance in bladder cancer tissues and cell lines. BMC Cancer. 2009;9:357.

16. Picco G, Julien S, Brockhausen I, Beatson R, Antonopoulos A, Haslam S, et al. Over-expression of ST3Gal-I promotes mammary tumorigenesis. Glycobiology. 2010;20(10):1241-50.

17. Burchell J, Poulsom R, Hanby A, Whitehouse C, Cooper L, Clausen H, et al. An alpha 2,3 sialyltransferase (ST3Gal I) is elevated in primary breast carcinomas. Glycobiology. 1999;9(12):1307-11.

18. Edge SB, Compton CC. The American Joint Committee on Cancer: the 7th Edition of the AJCC Cancer Staging Manual and the Future of TNM. Ann Surg Oncol. 2010;17(6):1471-4.

19. Leibovich BC, Blute ML, Cheville JC, Lohse CM, Frank I, Kwon ED, et al. Prediction of progression after radical nephrectomy for patients with clear cell renal cell carcinoma - A stratification tool for prospective clinical trials. Cancer-Am Cancer Soc. 2003;97(7):1663-71.

20. Hakomori S. Glycosylation defining cancer malignancy: New wine in an old bottle. Proc Natl Acad Sci U S A. 2002;99(16):10231-3.

21. Guo HB, Nairn A, Harris K, Randolph M, Alvarez-Manilla G, Moremen K, et al. Loss of expression of $\mathrm{N}$-acetylglucosaminyltransferase $\mathrm{Va}$ results in altered gene expression of glycosyltransferases and galectins. Febs Lett. 2008;582(4):527-35.

22. Paulson JC, Blixt O, Collins BE. Sweet spots in functional glycomics. Nat Chem Biol. 2006;2(5):238-48. 
23. Varki A, Schauer R. Sialic acids. In: Varki A, Cummings RD, Esko JD, Freeze $\mathrm{HH}$, Stanley P, Bertozzi CR, Hart GW, Etzler ME, editors. Essentials of glycobiology. 2nd ed. NY: Cold Spring Harbor; 2009.

24. Bull C, Stoel MA, den Brok MH, Adema GJ. Sialic acids sweeten a Tumor's life. Cancer Res. 2014;74(12):3199-204.

25. Sakuma K, Aoki M, Kannagi R. Transcription factors C-Myc and CDX2 mediate Eselectin ligand expression in colon cancer cells undergoing EGF/bFGF-induced epithelial-mesenchymal transition. Proc Natl Acad Sci U S A 2012;109(20):7776-81.

26. Duan LL, Guo P, Zhang Y, Chen HL. Regulation of metastasis-suppressive gene nm23-H1 on glycosyl-transferases involved in the synthesis of sialyl lewis antigens. J Cell Biochem. 2005;94(6):1248-57.

27. Nakamori S, Kameyama M, Imaoka S, Furukawa H, Ishikawa O, Sasaki Y, et al. Increased expression of sialyl lewis $(X)$ antigen correlates with poor survival in patients with colorectal-carcinoma - clinicopathological and immunohistochemical study. Cancer Res. 1993;53(15):3632-7.

28. Koike T, Kimura N, Miyazaki K, Yabuta T, Kumamoto K, Takenoshita S, et al. Hypoxia induces adhesion molecules on cancer cells: a missing link between Warburg effect and induction of selectin-ligand carbohydrates. Proc Natl Acad Sci U S A. 2004;101(21):8132-7.

29. Nakamori S, Kameyama M, Imaoka S, Furukawa H, Ishikawa O, Sasaki Y, et al. Involvement of carbohydrate antigen sialyl Lewis(X) in colorectal cancer metastasis. Dis Colon Rectum. 1997;40(4):420-31.

30. Krause T, Turner GA. Are selectins involved in metastasis? Clin Exp Metastas. 1999;17(3):183-92.

31. Borsig L, Wong R, Feramisco J, Nadeau DR, Varki NM, Varki A. Pictures in molecular medicine - three-dimensional visualization of intravascular tumor cells in mice. Trends Mol Med. 2001;7(8):377-7.

32. Fuster MM, Esko JD. The sweet and sour of cancer: glycans as novel therapeutic targets. Nat Rev Cancer. 2005;5(7):526-42.

33. Rillahan CD, Antonopoulos A, Lefort CT, Sonon R, Azadi P, Ley K, et al. Global metabolic inhibitors of sialyl- and fucosyltransferases remodel the glycome. Nat Chem Biol. 2012;8(7):661-8.

34. Bull C, Boltje TJ, Wassink M, de Graaf AMA, van Delft FL, den Brok MH, et al. Targeting aberrant sialylation in cancer cells using a fluorinated sialic acid analog impairs adhesion, migration, and in vivo tumor growth. Mol Cancer Ther. 2013;12(10):1935-46.

\section{Submit your next manuscript to BioMed Central and take full advantage of:}

- Convenient online submission

- Thorough peer review

- No space constraints or color figure charges

- Immediate publication on acceptance

- Inclusion in PubMed, CAS, Scopus and Google Scholar

- Research which is freely available for redistribution 